\title{
Foreign body in the abdomen
}

\author{
Wei-Jing Lee • Yu-Hui Lu • Si-Chun Vong • Tang-Yu Lin
}

Received: 28 March 2009/Accepted: 29 November 2009/Published online: 18 May 2010

(C) Springer-Verlag London Ltd 2009

A 4-year-old boy without any medical history of systemic disease was brought to the emergency department by his parent after accidentally swallowing a coin: one New Taiwan Dollar $(2 \mathrm{~cm} \times 2 \mathrm{~cm})$. An X-ray of the abdomen was arranged and showed a round-shaped metallic object over the left upper quadrant region which may possibly be located in the stomach (Fig. 1). Serial X-rays of the abdomen were done 3 days later, 1 week later and 2 weeks later on an outpatient basis with laxatives prescribed. The coin passed spontaneously roughly 28 days later without any need for surgical intervention.

Accidental foreign body ingestion in the gastrointestinal system occurs more frequently in childhood than in adulthood. Oral dental implants, bezoars, chicken/fish bones, packages of medications and drugs are among the other foreign bodies commonly seen to be swallowed [1] When dealing with the intestinal foreign body ingestion, location and the characteristics of the foreign body have a great impact in terms of management. Early endoscopic intervention, in general, may be required and applied for objects wider than $2 \mathrm{~cm}$ and longer than $5 \mathrm{~cm}$, since their passage through the pylorus and duodenum will become

W.-J. Lee $\cdot$ S.-C. Vong $\cdot$ T.-Y. Lin $(\bowtie)$

Department of Emergency Medicine, Chi-Mei Medical Center, 901 Chung-Hua Road,

Yung-Kang City, Tainan 710, Taiwan

e-mail: weijing1103@gmail.com

Y.-H. Lu

Department of Emergency Medicine, Chi-Mei Medical Center Liou-Ying Branch,

Tainan, Taiwan

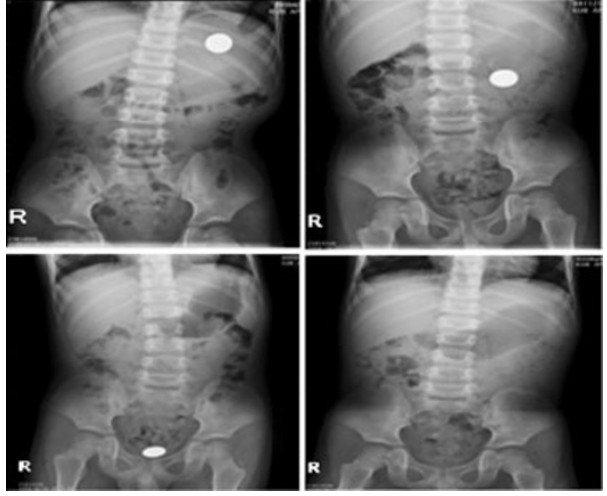

Fig. 1 Serial radiographs of the abdomen showingspontaneous passage of a round metallic object 28 days later

rather difficult [2]. Once the foreign body passes beyond the pylorus and ileocaecal valve, it will be easily removed out of the lumen [2]. Intestinal foreign body retention will not reveal any symptoms as long as the object does not cause any intestinal obstruction or perforation. The patient should be monitored on outpatient policlinic follow-up and be informed to return to the hospital in case of acute abdominal pain caused by intestinal obstruction or perforation.

\section{References}

1. Wade RG, Robert AB (2004) Swallowed foreign bodies. In: Marx JA, Hockberger RS, Walls RM (eds) Rosen's emergency medicine, 6th edn. Elsevier Health Sciences, Philadelphia, pp 513-516

2. Eryilmaz M, Ozturk O, Mentes O et al (2006) Intracolonic multiple pebbles in young adults: radiographic imaging and conventional approach to a case. World J Gastroenterol 12(37):6074-6076 